\title{
Rephine.r: a pipeline for correcting gene calls and clusters to improve phage pangenomes and phylogenies
}

\author{
Jason W Shapiro ${ }^{\text {Corresp., } 1}$, Catherine Putonti ${ }^{1,2,3}$ \\ 1 Department of Biology, Loyola University Chicago, Chicago, Illinois, United States \\ 2 Department of Microbiology and Immunology, Stritch School of Medicine, Loyola University Chicago, Maywood, Illinois, United States \\ 3 Bioinformatics Program, Loyola University Chicago, Chicago, Illinois, United States \\ Corresponding Author: Jason W Shapiro \\ Email address: jshapiro2@luc.edu
}

Background. A pangenome is the collection of all genes found in a set of related genomes. For microbes, these genomes are often different strains of the same species, and the pangenome offers a means to compare gene content variation with differences in phenotypes, ecology, and phylogenetic relatedness. Though most frequently applied to bacteria, there is growing interest in adapting pangenome analysis to bacteriophages. However, working with phage genomes presents new challenges. First, most phage families are under-sampled, and homologous genes in related viruses can be difficult to identify. Second, homing endonucleases and intron-like sequences may be present, resulting in fragmented gene calls. Each of these issues can reduce the accuracy of standard pangenome analysis tools.

Methods. We developed an R pipeline called Rephine.r that takes as input the gene clusters produced by an initial pangenomics workflow. Rephine.r then proceeds in two primary steps. First, it identifies three common causes of fragmented gene calls: 1 ) indels creating early stop codons and new start codons; 2) interruption by a selfish genetic element; and 3) splitting at the ends of the reported genome. Fragmented genes are then fused to create new sequence alignments. In tandem, Rephine.r searches for distant homologs separated into different gene families using Hidden Markov Models. Significant hits are used to merge families into larger clusters. A final round of fragment identification is then run, and results may be used to infer single-copy core genomes and phylogenetic trees.

Results. We applied Rephine.r to three well-studied phage groups: the Tevenvirinae (e.g. T4), the Studiervirinae (e.g. T7), and the Pbunaviruses (e.g. PB1). In each case, Rephine.r recovered additional members of the single-copy core genome and increased the overall bootstrap support of the phylogeny. The Rephine.r pipeline is provided through GitHub ( https://www.github.com/coevoeco/Rephine.r ) as a single script for automated analysis and with utility functions to assist in building single-copy core genomes and predicting the sources of fragmented genes. 
1 Rephine.r: a pipeline for correcting gene calls and clusters to improve phage

2 pangenomes and phylogenies

3

4 Jason W. Shapiro ${ }^{1}$, Catherine Putonti ${ }^{1,2,3}$

5

61 Department of Biology, Loyola University Chicago, Chicago, Illinois, USA

$7 \quad 2$ Department of Microbiology and Immunology, Stritch School of Medicine, Loyola

8 University Chicago, Maywood, Illinois, USA

$9 \quad{ }^{3}$ Bioinformatics Program, Loyola University Chicago, Chicago, Illinois, USA

12 Corresponding Author:

13 Jason Shapiro ${ }^{1}$

141032 W Sheridan Rd, Chicago, IL, 60660, USA

15 Email address: jshapiro2@luc.edu 


\section{Abstract}

18 Background. A pangenome is the collection of all genes found in a set of related genomes. For microbes, these genomes are often different strains of the same species, and the pangenome offers a means to compare gene content variation with differences in phenotypes, ecology, and phylogenetic relatedness. Though most frequently applied to bacteria, there is growing interest in adapting pangenome analysis to bacteriophages. However, working with phage genomes presents new challenges. First, most phage families are under-sampled, and homologous genes in related viruses can be difficult to identify. Second, homing endonucleases and intron-like sequences may be present, resulting in fragmented gene calls. Each of these issues can reduce the accuracy of standard pangenome analysis tools.

Methods. We developed an R pipeline called Rephine.r that takes as input the gene clusters produced by an initial pangenomics workflow. Rephine.r then proceeds in two primary steps. First, it identifies three common causes of fragmented gene calls: 1) indels creating early stop codons and new start codons; 2 ) interruption by a selfish genetic element; and 3) splitting at the ends of the reported genome. Fragmented genes are then fused to create new sequence alignments. In tandem, Rephine.r searches for distant homologs separated into different gene families using Hidden Markov Models. Significant hits are used to merge families into larger clusters. A final round of fragment identification is then run, and results may be used to infer single-copy core genomes and phylogenetic trees.

Results. We applied Rephine.r to three well-studied phage groups: the Tevenvirinae (e.g. T4), the Studiervirinae (e.g. T7), and the Pbunaviruses (e.g. PB1). In each case, Rephine.r recovered additional members of the single-copy core genome and increased the overall bootstrap support of the phylogeny. The Rephine.r pipeline is provided through GitHub (https://www.github.com/coevoeco/Rephine.r) as a single script for automated analysis and with utility functions to assist in building single-copy core genomes and predicting the sources of fragmented genes. 


\section{Introduction}

49 A pangenome is the collection of all genes found in a set of related genomes (Tettelin et al., 2005; Vernikos et al., 2015). These genomes might be different strains of the same species or taken from the same genus or higher taxonomic level. Pangenomes are useful, because they allow one to compare gene content variation to differences in phenotypes, ecology, and evolutionary history. For instance, by mapping gene content of potential pathogens onto a phylogeny and contrasting clade-specific genes with differences in reported strain virulence, the pangenome can help reveal how these genes relate to pathogenicity while placing them in an evolutionary context (e.g. (Hurtado et al., 2018; Wyres et al., 2019)). Pangenomes have also been used to describe which functions are conserved among members of bacterial taxa in different environments (e.g. (Zhang \& Sievert, 2014)).

Pangenome analysis is most commonly applied to bacteria. Due to the explosion of data from metagenomes and microbiome studies, many bacterial taxa are wellsampled and can be associated with large sets of ecological or health-related metadata. Additionally, multiple software packages are available that facilitate automated inference of bacterial pangenomes, such as Anvi'o (Eren et al., 2021) and Roary (Page et al., 2015).

A typical pangenome analysis pipeline starts with two main steps: gene prediction and gene clustering. Often, workflows also include subsequent steps for function prediction, sequence alignment, and core gene identification. The accuracy of the two primary steps of inferring a pangenome is paramount. If a gene caller ignores an open reading frame (ORF) or inaccurately returns the end position of the ORF, genes may be truncated or merged. Errors in clustering-the process of placing related sequences into gene families - can include grouping unrelated genes or failing to place homologs in the same cluster. Together, these errors in gene calling and clustering may significantly impact identification of the "single-copy core genome" (SCG). The SCG is commonly used as the basis for phylogenetic inference, and excluding genes can mean missing important sequence variation and building less informative trees.

There is growing interest in applying pangenomic and phylogenomic workflows to bacteriophages (e.g. (Edwards et al., 2019; Bellas et al., 2020)). Just as the deluge of 
79

80

81

82

83

84

85

86

87

88

89

90

91

92

93

94

95

96

97

98

99

100

101

102

103

104

105

106

107

108

109

metagenomic data has expanded bacterial comparative genomics, thousands of phage genomes are now published every year (Roux et al., 2019; Dion, Oechslin \& Moineau, 2020). Because no single gene is conserved among all phage genomes, gene content profiles and gene sharing networks have become standard tools in virus taxonomy for identifying and comparing related viruses (Bolduc et al., 2017; Shapiro \& Putonti, 2018).

In the process, pangenomics has become an intrinsic component of phage bioinformatics.

Many of the potential sources of error for bacterial pangenome analysis are amplified when studying phages. First, phages are under-sampled despite regular publication of new genomes and identification of prophages within bacterial genomes (Dion, Oechslin \& Moineau, 2020). Isolation, even of better-sampled groups through dedicated programs like SEA-PHAGES continues to discover novel viruses with genes lacking obvious homology to any known sequence (Pope et al., 2015). As a result, we often try to compare virus genomes that are more distantly related than expected for most pangenomic workflows. This can make it difficult to recognize homologs between phage genomes that have low sequence identity. Further, many phages include intronlike sequences and homing endonucleases (Belfort, 1990; Stoddard, 2005). These selfish genetic elements interrupt genes and cause fragmented gene calls during annotation. Thus, the two main tasks of a pangenome analysis-gene identification and gene clustering-are more error-prone with phages than with bacteria.

Here, we describe a pipeline implemented in $R$, Rephine.r, for identifying and correcting common errors in the initial gene clusters and gene calls returned by pangenomic workflows. Given the results from a traditional pangenome analysis, Rephine.r: 1) merges gene clusters using Hidden Markov Models (HMMs) and 2) identifies fragmented gene calls to avoid the overprediction of paralogs and to improve sequence alignments. Each of the steps in Rephine.r can also be run separately for individual use cases that require only cluster merging or defragmentation. We demonstrate the value of Rephine.r using three phage taxa: the Tevenvirinae (e.g. T4), the Studiervirinae (e.g. T7), and the Pbunaviruses (e.g. PB1). These virus groups represent a range of genome sizes and sampling depth, and each has at least 30 members with a RefSeq assembly. We show that correcting errors in gene cluster and 
110 gene fragmentation increases the size of the SCG in each case and enables inference

111 of better-supported phylogenies. The tool is available through GitHub as a command

112 line R script (https://www.github.com/coevoeco/Rephine.r) and includes utility scripts for

113 returning the single-copy core genes and classifying the causes of gene fragmentation

114 events.

115

116 Materials \& Methods

117 Overview of the pipeline

118 The Rephine.r pipeline (summarized in Fig. 1) assumes the researcher has already

119 completed a workflow for predicting gene clusters in a pangenome, such as the

120 combination of blastp (Altschul et al., 1990) and MCL (Enright, Van Dongen \&

121 Ouzounis, 2002) implemented by Anvi'o (Eren et al., 2021) and other programs (e.g.

122 vConTACT (Bolduc et al., 2017) and Roary (Page et al., 2015)). In what follows, we use

123 Anvi'o as the basis for initial pangenomes, as Anvi'o is both a popular tool for bacterial

124 pangenomes and includes several useful commands for facilitating our corrections.

125 Future updates will expand Rephine.r's compatibility with other tools.

126 Following initial gene clustering, the Rephine.r pipeline: 1) identifies and merges

127 gene clusters containing distantly related homologs using HMMs, and 2) identifies

128 fragmented gene calls that can be fused for the purpose of SCG inference and

129 generating phylogenies. By default, Rephine.r will first run the cluster merging and

130 defragmentation steps in tandem, produce a set of new clusters that combine the

131 results of these corrections, and will then run a second round of defragmentation to

132 identify any new cases that emerge due to the prior steps. Command line options are

133 also offered for users that wish to run the HMM merging or fragment fusion steps

134 individually. In addition to the main pipeline, we include two complementary scripts:

135 getSCG.r, returns the single-copy core genes and a concatenated alignment file for

136 phylogenetics; fragclass.r categorizes the likely events that led to fragmented gene

137 calls.

138

Merging gene families with HMMs 
140 Gene clustering based on sequence similarity relies on threshold criteria for defining 141 when two sequences are related and for clustering related sequences into groups. In 142 Anvi'o, the default identity heuristic is defined by the "minbit" score, the ratio of the 143 BLAST bit score between two sequences and the minimum bit score from blasting each 144 sequence against itself. This metric generally performs well, and for bacteria, where 145 homologs are typically over $50 \%$ identical, it is especially successful. For phages, 146 however, this approach can miss more distant homologs. Even using a 35\% amino acid 147 identity threshold (Cresawn et al., 2011; Shapiro \& Putonti, 2018), we may miss cases 148 that only appear related when viewing alignments or comparing phage genes by 149 structure or synteny. Unfortunately, it is not as simple as specifying a lower minbit 150 threshold, since doing so will also increase the number of unrelated genes that are 151 clustered together erroneously.

152 Given the initial gene clusters returned by Anvi'o, Rephine.r builds separate 153 HMM profiles for each cluster using the hmmbuild function from HMMER (Eddy, 1998) and converts the concatenated HMM profiles into a database with hmmpress. The script then uses hmmscan to compare every original gene call against each HMM profile. This step is expected to be more sensitive for recognizing distant homologs than the initial blastp, as the HMM profiles make use of variation from multiple members of the same cluster. Significant hits are then defined as follows: for each original gene cluster, the "minimum self-bit" (or "selfbit") score is recorded as the minimum of the bit scores for each of the gene calls that was initially assigned to that cluster by MCL. This selfbit score then serves as a profile-specific significance threshold. Any gene call that was originally assigned to another cluster but has a bit score greater than this value is then used to establish a putative connection between gene clusters. We also include the option of specifying an absolute minimum bit score as an additional criterion. These connections are recorded in the form of a network edgelist linking gene calls to gene clusters. Next, this edgelist is relabeled to define edges between the original gene clusters that share putative homologs. Finally, this edgelist is used to generate a network with the R (R Core Team, 2013) package igraph (Csardi, Nepusz \& Others, 2006), and the connected components are returned with the function "components". The 
170 result defines sets of the original gene clusters that are suitable for merging into a

171 single, larger cluster.

172

\section{Identifying fragmented gene calls}

174 To find fragmented genes, Rephine.r first identifies every gene cluster that includes at 175 least two sequences from the same genome. These sequences may represent true 176 duplicates or paralogs, or they may be separate pieces of the same original sequence 177 that have been split by one of several processes, including: a frameshift due to an indel, 178 insertion of a selfish genetic element, or being artificially split across the ends of the 179 genome when it was reported to GenBank. This third case may also arise as an artifact 180 of the two other mechanisms. For any of these scenarios, the two pieces of the gene 181 will be notable in two ways: 1) they will align with separate parts of the gene in a multiple sequence alignment, with one piece corresponding to an N-terminal fragment, and the other to the C-terminus; 2) they should have lower sequence similarity to each other than to the average comparison with other sequences in the multiple sequence alignment. Fig. 2a illustrates how a fragmented gene may appear in an alignment.

Given clusters with potential fragments, every gene call within an affected cluster is compared using blastp to every other gene call in the same cluster. For the two focal gene calls from a potential fragmented gene, the bit score from their blast alignment is compared to the mean bit score for other blast results within the gene cluster. We defined the ratio of this pairwise blast to the cluster average as the "relative bit" (or "relbit"). Mathematically, for potential fragments A and B within a gene cluster $G$, this is defined as:

$194 \operatorname{relbit}(A, B)=\frac{\operatorname{bit}(A, B)}{\overline{\operatorname{bit}}(A, G)}, \operatorname{relbit}(B, A)=\frac{\operatorname{bit}(B, A)}{\overline{\operatorname{bit}}(B, G)}$ Eqn. 1 where the overbar refers to the mean. The maximum of these relbit values is then used as a criterion for judging similarity between $A$ and $B$. If this value is below a chosen threshold, the ORFs are considered to be sufficiently dissimilar. 
199

200

201

202

203

204

205

206

207

208

209

210

211

212

213

214

215

216

217

218

219

220

221

222

223

224

225

226

227

228

21

Rephine.r also compares the extent of overlap within the pairwise alignment space between each potential paralog. This step is needed, because dissimilar gene fragments may still have overlaps in the alignment due to alignment errors or if the original fragmentation event was caused by a short duplication. To quantify this overlap, the "percent overlap" is calculated as the size of the ORFs' intersection within the alignment divided by the number of unique, aligned positions between the two sequences. In mathematical terms, for a gene with potential fragments $A$ and $B$, we define:
Percent Overlap $=\frac{|A \cap B|}{|A \cup B|}$
Eqn. 2

where the size terms are based solely on the aligned positions within the multiple sequence alignment.

Ultimately, sequence pairs with low relative bit scores ("relbit") and low percent overlaps ("percoverlap") are the likeliest to fit our expectations of a fragmented gene call. In practice, we implemented default parameters for these criteria of 0.25 for "relbit" and 0.25 for "percoverlap." These choices are based on plotting values of each parameter (Supplemental Fig. 1) from the test cases described below and identifying a set of points that weakly cluster together in the graph. When checked manually, each of these genes appeared to correspond to fragmented calls, whereas nearby points in the graph included potential errors. These parameters can be adjusted at the command line, and we would encourage others to visually inspect their alignments.

Once fragmented genes are identified, a new FASTA file is created in which the original pieces of the full-length gene are artificially spliced (or "fused") into a single gene call. To preserve the original event that separated the sequences, the script inserts an " $X$ " between the two pieces of the gene. New alignments are then made with MUSCLE (Edgar, 2004) for each affected gene cluster, with these X's imposing a gap in the alignment (see Fig. $2 b$ for an illustration of this step). If desired, the user can then use the additional script, getSCG.r, to return a list of the single-copy core gene clusters, along with a concatenated alignment file that is suitable for phylogenetics. The script, 
229 fragclass.r, can also be used to obtain a table summarizing predicted causes for each 230 type of fragment based on the separation between the original gene calls.

231

232 Virus genomic data

233 Phages in the subfamily Studiervirinae (family Autographiviridae), the subfamily

234 Tevenvirinae (family Myoviridae), and the genus Pbunavirus (family Myoviridae) were 235 chosen as well-studied examples for testing Rephine.r. We downloaded all available 236 RefSeq genomes from each of these taxa from the National Center for Biotechnology 237 Information's (NCBI) genome browser (as of February 2021). This data set included 145 238 Studierviruses, 127 Tevenviruses, and 38 Pbunaviruses (a full list of accessions is 239 included in Supplementary Table 1). The Studiervirinae (e.g. phages T3 and T7) and 240 the Tevenvirinae (e.g. phage T4) are among the best-studied phage subfamilies and 241 include characterized examples of introns and homing endonucleases (Chu et al., 1986; 242 Belle, Landthaler \& Shub, 2002; Bonocora \& Shub, 2004; Petrov, Ratnayaka \& Karam, 243 2010). These features made these two subfamilies ideal for testing methods for 244 identifying distant homologs and fragmented gene calls. The Pbunaviruses were chosen 245 due to the relatively large number of available genomes at the genus level, offering a 246 less diverse contrast to the other phage groups.

247

Initial pangenome workflow with Anvi'o

249 We built an initial pangenome for each phage group using Anvi'o v6.2 (Eren et al., 250 2021) following the standard pangenomics workflow

251

252 253

254

(https://merenlab.org/2016/11/08/pangenomics-v2/), which uses Prodigal (Hyatt et al., 2010) for gene calling. Alternative gene callers can also be used, and these gene calls can be imported into Anvi'o as part of the anvi-gen-contigs-database program The "-use-ncbi-blast" flag was specified for the anvi-pan-genome command. Due to the large genetic diversity of phages, we set the minbit threshold to 0.35 , based on prior work (Cresawn et al., 2011; Shapiro \& Putonti, 2018).

\section{Phylogenetics}


259 Maximum likelihood phylogenies were estimated using IQTREE v2.0.3 (Nguyen et al., 260 2015) with ModelFinder (Kalyaanamoorthy et al., 2017) to automate choosing the 261 optimal substitution model for each tree. For each of the three virus groups, trees were 262 built based on concatenated alignments for the original SCGs and again following 263 Rephine.r using the expanded SCGs. Tree summary statistics were computed in R 264 using the ape package (Paradis, Claude \& Strimmer, 2004) and drawn using ggtree (Yu 265 et al., 2017).

266

267

Code Availability

268

All code for this work is provided on GitHub

269

(https://github.com/coevoeco/Rephine.r). The code includes a walkthrough for running

270 Rephine.r following a standard Anvi'o workflow, as well as utility scripts, getSCG.r and

271 fragclass.r, that provide additional output of the SCG genes and predicted causes of 272 fragmentation events.

273

274

\section{Results}

275

To test the Rephine.r pipeline, we downloaded all available RefSeq genomes for the 276 Studiervirinae, Tevenvirinae, and Pbunaviruses from NCBI. We then followed the 277 standard pangenomic workflow for Anvi'o to facilitate initial MCL clustering based on blastp scores. Results and basic information about these taxa are summarized in Table 1. Across all Studierviruses, there were only 12 core genes, of which three were singlecopy. Tevenviruses included 27 core genes (13 single-copy), and the Pbunaviruses had 28 core genes (19 single-copy).

We ran Rephine.r with default settings, which first predicts fragmented gene calls within each gene cluster. In tandem, it identifies related gene clusters using HMMs. It then combines the results from these steps to produce new merged clusters with corrections for fragmented genes. Last, it runs a second defragmentation step to identify instances where fragmented gene calls were originally split into separate gene clusters. We examined results to see how the core genome changed after each step and how the final SCG affected phylogenetic inference. 
The initial HMM merging step resulted in two additional core genes for

290

291

292

293

294

295

296

297

298

299

300

301

302

303

304

305

306

307

308

309

310

311

312

313

314

315 Studierviruses and 10 additional core genes for Tevenviruses but no new single-copy core genes for any of the virus groups. Notably, several mergers involved more than two gene clusters. In one case for the Tevenvirinae, 30 separate gene clusters were merged, corresponding to the phage tail fiber. Defragmenting gene calls expanded the SCG for each taxon, increasing the Studiervirinae SCG to 8 genes, the Tevenvirinae to 22 genes, and the Pbunaviruses to 26 genes (all but two of the Pbunavirus core genes). The final round of defragmentation identified additional fragmented genes but no additional core genes.

We then built phylogenies for each taxon with the original SCGs and with expanded SCGs following Rephine.r. With only three single-copy core genes, the initial Studiervirinae tree contained multiple unresolved polytomies and branches with poor support (Fig. 3a). The updated tree based on eight genes had improved overall bootstrap support and displayed greater resolution of closely related genomes (Fig. 3b). (A version of the tree with all labels is provided in Supplemental Fig. 2.) Trees for the Tevenvirinae and Pbunaviruses (Supplemental Fig. 3 and Supplemental Fig. 4) also had improved bootstrap support. In the case of the Pbunaviruses, the tree remains poorly resolved with very short branches, despite being built from the most genes, as there was insufficient variation among the viruses from this genus.

Last, we checked the results from gene call defragmentation for known instances of introns and homing endonucleases in the Studiervirinae and Tevenvirinae. These include interruptions to DNA polymerase in members of Studiervirinae (Bonocora \& Shub, 2004) and Tevenvirinae (Petrov, Ratnayaka \& Karam, 2010) and thymidylate synthase in T4 (Chu et al., 1986). After running Rephine.r, we identified a single-copy core gene that corresponded to each gene of interest. In each case, inclusion in the SCG was only possible after fragment identification.

\section{Discussion}

317 We describe Rephine.r, a pipeline for improving results of phage pangenome analysis 318 by merging gene clusters containing distant homologs and correcting gene calls that have been fragmented or interrupted by selfish genetic elements. Using the 
320 Tevenvirinae, Studiervirinae, and Pbunaviruses as test cases, we show how this 321 process expands the putative SCG for each group, enabling more accurate estimates of 322 gene conservation. For the Tevenvirinae and Studiervirinae, this also improved the 323 quality of the phylogenies, whereas for Pbunaviruses there was still insufficient variation 324 among the genomes to produce a reliable tree.

325 The present work provides a first step for expanding the usage of phylogenetics

326

327 328 329 with diverse phage genomes. A key concept that we include (which we took advantage of using manual corrections previously (Shapiro \& Putonti, 2020)) is the use of artificially spliced sequences following the identification of interrupted genes. This type of correction is unsurprising when working with eukaryotic exons, but it is generally ignored with microbes, because we often fail to appreciate that intron-like sequences are common features of many phages. Biologically, it is uncertain how often these interrupted genes remain functional or if the separated ORFs correspond to separate functions. However, several studies report fully functional, single protein products for phage genes separated by introns (Belfort, 1990) or inteins (Kelley et al., 2016), as well as at least one case where a gene split by a homing endonuclease remains active (Friedrich et al., 2007). Though these ORFs may be interrupted by over 1000 nucleotides, these interruptions likely correspond to a single mutational event, and the ORFs should still be treated as a single gene when reconstructing the SCG and an associated phylogeny. In both the Studiervirinae and the Tevenvirinae, our approach accurately recognized known homing endonucleases and introns, and these genes themselves remain the most common multi-copy core genes following Rephine.r. How interrupted genes are interpreted in functional genomics studies is an important question, and these fragmented genes should be treated with additional care when reporting the functional repertoire of genomes.

It is important to note that we have focused our application of Rephine.r on test cases involving single-contig, RefSeq assemblies. In the case of draft genome assemblies comprised of multiple contigs (less common for phages under $100 \mathrm{~kb}$ ), we expect to observe instances where a gene call is separated into different ORFs on different contigs. These errors will result in overestimating gene content and incorrect predictions of paralogous sequences. Similar issues have been noted to cause errors in 
351 the analysis of gene content evolution in eukaryotes (Denton et al., 2014). The current

352 implementation of gene defragmentation in Rephine.r should successfully resolve many

353 of these mistakes, and it may offer a future approach for consolidating contigs in

354 assemblies. For instance, suppose a gene is split by a transposase that includes short

355 palindromic repeats. These regions are difficult to assemble with short reads and may

356 lead to one contig ending with half of the original gene, while a second contig starts with

357 the transposase and the remainder of the gene. Scaffolding these contigs can be

358 challenging, but by recognizing gene fragments, it may be possible to resolve the 359 assembly.

360 Last, bacterial pangenome workflows typically do not account for specific issues

361 that may arise for prophage regions, such as errors in clustering and gene

362 fragmentation that we observe in the genomes of phage isolates. Our expectation is that

363 these same errors will impact prophages, and future work will need to consider how

364 these issues may impact the accuracy of bacterial pangenomes. Moreover, bacterial

365

genes themselves can be interrupted by mobile genetic elements (including phages),

366

and Rephine.r should offer a novel approach for identifying these events.

367

\section{Conclusions}

369 The Rephine.r pipeline offers an efficient means to identify and correct errors in phage 370 pangenomes caused by incomplete gene clustering and fragmented gene calls.

371 Correcting these errors, in particular for cases of genes interrupted by selfish genetic

372 elements, increases the size of the SCG in each of our test cases. These corrections

373 provide more genetic variation for improved phylogenetic inference and are especially

374 useful for large, diverse phage groups where standard methods produce limited core

375 genomes and poorly resolved phylogenies.

376

377 Acknowledgements

378 We are grateful to the members of the Putonti Lab for feedback on this work.

379

\section{References}


381 Altschul SF, Gish W, Miller W, Myers EW, Lipman DJ. 1990. Basic local alignment 382 search tool. Journal of molecular biology 215:403-410.

383 Belfort M. 1990. Phage T4 introns: self-splicing and mobility. Annual review of genetics 384 24:363-385.

Bellas CM, Schroeder DC, Edwards A, Barker G, Anesio AM. 2020. Flexible genes establish widespread bacteriophage pan-genomes in cryoconite hole ecosystems. Nature communications 11:4403.

Belle A, Landthaler M, Shub DA. 2002. Intronless homing: site-specific endonuclease 389 SegF of bacteriophage T4 mediates localized marker exclusion analogous to homing endonucleases of group I introns. Genes \& development 16:351-362.

Bolduc B, Jang HB, Doulcier G, You Z-Q, Roux S, Sullivan MB. 2017. vConTACT: an 392 iVirus tool to classify double-stranded DNA viruses that infect Archaea and Bacteria. PeerJ 5:e3243.

394 395 396 397

Bonocora RP, Shub DA. 2004. A self-splicing group I intron in DNA polymerase genes of T7-like bacteriophages. Journal of bacteriology 186:8153-8155.

Chu FK, Maley GF, West DK, Belfort M, Maley F. 1986. Characterization of the intron in the phage T4 thymidylate synthase gene and evidence for its self-excision from the primary transcript. Cell 45:157-166.

Cresawn SG, Bogel M, Day N, Jacobs-Sera D, Hendrix RW, Hatfull GF. 2011. Phamerator: a bioinformatic tool for comparative bacteriophage genomics. $B M C$ bioinformatics 12:395.

Csardi G, Nepusz T, Others. 2006. The igraph software package for complex network research. InterJournal, complex systems 1695:1-9. 
404 Denton JF, Lugo-Martinez J, Tucker AE, Schrider DR, Warren WC, Hahn MW. 2014.

405 Extensive error in the number of genes inferred from draft genome assemblies.

$406 \quad$ PLoS computational biology 10:e1003998.

407 Dion MB, Oechslin F, Moineau S. 2020. Phage diversity, genomics and phylogeny.

$408 \quad$ Nature reviews. Microbiology 18:125-138.

409 Eddy SR. 1998. Profile hidden Markov models. Bioinformatics 14:755-763.

410 Edgar RC. 2004. MUSCLE: multiple sequence alignment with high accuracy and high throughput. Nucleic acids research 32:1792-1797.

412 Edwards RA, Vega AA, Norman HM, Ohaeri M, Levi K, Dinsdale EA, Cinek O, Aziz RK, McNair K, Barr JJ, Bibby K, Brouns SJJ, Cazares A, de Jonge PA, Desnues C, Díaz Muñoz SL, Fineran PC, Kurilshikov A, Lavigne R, Mazankova K, McCarthy DT, Nobrega FL, Reyes Muñoz A, Tapia G, Trefault N, Tyakht AV, Vinuesa P, Wagemans J, Zhernakova A, Aarestrup FM, Ahmadov G, Alassaf A, Anton J, Asangba A, Billings EK, Cantu VA, Carlton JM, Cazares D, Cho G-S, Condeff T, Cortés P, Cranfield M, Cuevas DA, De la Iglesia R, Decewicz P, Doane MP, Dominy NJ, Dziewit L, Elwasila BM, Eren AM, Franz C, Fu J, Garcia-Aljaro C, Ghedin E, Gulino KM, Haggerty JM, Head SR, Hendriksen RS, Hill C, Hyöty H, Ilina EN, Irwin MT, Jeffries TC, Jofre J, Junge RE, Kelley ST, Khan Mirzaei M, Kowalewski M, Kumaresan D, Leigh SR, Lipson D, Lisitsyna ES, Llagostera M, Maritz JM, Marr LC, McCann A, Molshanski-Mor S, Monteiro S, Moreira-Grez B, Morris M, Mugisha L, Muniesa M, Neve H, Nguyen N-P, Nigro OD, Nilsson AS, O'Connell T, Odeh R, Oliver A, Piuri M, Prussin AJ Ii, Qimron U, Quan Z-X, Rainetova P, Ramírez-Rojas A, Raya R, Reasor K, Rice GAO, Rossi A, Santos 
427

428

429

430

431

432

433

434

435

436

437

438

439

440

441

442

443

444

445

446

447

R, Shimashita J, Stachler EN, Stene LC, Strain R, Stumpf R, Torres PJ, Twaddle A, Ugochi Ibekwe M, Villagra N, Wandro S, White B, Whiteley A, Whiteson KL, Wijmenga C, Zambrano MM, Zschach H, Dutilh BE. 2019. Global phylogeography and ancient evolution of the widespread human gut virus crAssphage. Nature microbiology 4:1727-1736.

Enright AJ, Van Dongen S, Ouzounis CA. 2002. An efficient algorithm for large-scale detection of protein families. Nucleic acids research 30:1575-1584.

Eren AM, Kiefl E, Shaiber A, Veseli I, Miller SE, Schechter MS, Fink I, Pan JN, Yousef M, Fogarty EC, Trigodet F, Watson AR, Esen ÖC, Moore RM, Clayssen Q, Lee MD, Kivenson V, Graham ED, Merrill BD, Karkman A, Blankenberg D, Eppley JM, Sjödin A, Scott JJ, Vázquez-Campos X, McKay LJ, McDaniel EA, Stevens SLR, Anderson RE, Fuessel J, Fernandez-Guerra A, Maignien L, Delmont TO, Willis AD. 2021. Community-led, integrated, reproducible multi-omics with anvi'o. Nature microbiology 6:3-6.

Friedrich NC, Torrents E, Gibb EA, Sahlin M, Sjöberg B-M, Edgell DR. 2007. Insertion of a homing endonuclease creates a genes-in-pieces ribonucleotide reductase that retains function. Proceedings of the National Academy of Sciences of the United States of America 104:6176-6181.

Hurtado R, Carhuaricra D, Soares S, Viana MVC, Azevedo V, Maturrano L, Aburjaile F. 2018. Pan-genomic approach shows insight of genetic divergence and pathogenic-adaptation of Pasteurella multocida. Gene 670:193-206. 
448 Hyatt D, Chen G-L, Locascio PF, Land ML, Larimer FW, Hauser LJ. 2010. Prodigal: 449 prokaryotic gene recognition and translation initiation site identification. BMC bioinformatics 11:119.

451

452

Kalyaanamoorthy S, Minh BQ, Wong TKF, von Haeseler A, Jermiin LS. 2017. ModelFinder: fast model selection for accurate phylogenetic estimates. Nature methods 14:587-589.

Kelley DS, Lennon CW, SEA-PHAGES, Belfort M, Novikova O. 2016. Mycobacteriophages as Incubators for Intein Dissemination and Evolution. mBio 7. DOI: $10.1128 / \mathrm{mBio} .01537-16$.

Nguyen L-T, Schmidt HA, von Haeseler A, Minh BQ. 2015. IQ-TREE: a fast and effective stochastic algorithm for estimating maximum-likelihood phylogenies. Molecular biology and evolution 32:268-274.

Page AJ, Cummins CA, Hunt M, Wong VK, Reuter S, Holden MTG, Fookes M, Falush D, Keane JA, Parkhill J. 2015. Roary: rapid large-scale prokaryote pan genome analysis. Bioinformatics 31:3691-3693.

Paradis E, Claude J, Strimmer K. 2004. APE: Analyses of Phylogenetics and Evolution in R language. Bioinformatics 20:289-290.

Petrov VM, Ratnayaka S, Karam JD. 2010. Genetic insertions and diversification of the PolB-type DNA polymerase (gp43) of T4-related phages. Journal of molecular biology 395:457-474.

Pope WH, Bowman CA, Russell DA, Jacobs-Sera D, Asai DJ, Cresawn SG, Jacobs WR, Hendrix RW, Lawrence JG, Hatfull GF, Science Education Alliance Phage Hunters Advancing Genomics and Evolutionary Science, Phage Hunters 
471

472

473

474

475

476

477

478

479

480

481

482

483

484

485

486

487

488

489

490

491

492

493

Integrating Research and Education, Mycobacterial Genetics Course. 2015.

Whole genome comparison of a large collection of mycobacteriophages reveals a continuum of phage genetic diversity. eLife 4:e06416.

R Core Team. 2013. R: A language and environment for statistical computing.

Roux S, Adriaenssens EM, Dutilh BE, Koonin EV, Kropinski AM, Krupovic M, Kuhn JH, Lavigne R, Brister JR, Varsani A, Amid C, Aziz RK, Bordenstein SR, Bork P, Breitbart M, Cochrane GR, Daly RA, Desnues C, Duhaime MB, Emerson JB, Enault F, Fuhrman JA, Hingamp P, Hugenholtz P, Hurwitz BL, Ivanova NN, Labonté JM, Lee K-B, Malmstrom RR, Martinez-Garcia M, Mizrachi IK, Ogata H, Páez-Espino D, Petit M-A, Putonti C, Rattei T, Reyes A, Rodriguez-Valera F, Rosario K, Schriml L, Schulz F, Steward GF, Sullivan MB, Sunagawa S, Suttle CA, Temperton B, Tringe SG, Thurber RV, Webster NS, Whiteson KL, Wilhelm SW, Wommack KE, Woyke T, Wrighton KC, Yilmaz P, Yoshida T, Young MJ, Yutin N, Allen LZ, Kyrpides NC, Eloe-Fadrosh EA. 2019. Minimum Information about an Uncultivated Virus Genome (MIUViG). Nature biotechnology 37:29-37.

Shapiro JW, Putonti C. 2018. Gene Co-occurrence Networks Reflect Bacteriophage Ecology and Evolution. mBio 9. DOI: 10.1128/mBio.01870-17.

Shapiro JW, Putonti C. 2020. UPФ phages, a new group of filamentous phages found in several members of Enterobacteriales. Virus evolution 6:veaa030.

Stoddard BL. 2005. Homing endonuclease structure and function. Quarterly reviews of biophysics 38:49-95.

Tettelin H, Masignani V, Cieslewicz MJ, Donati C, Medini D, Ward NL, Angiuoli SV, Crabtree J, Jones AL, Scott Durkin A, DeBoy RT, Davidsen TM, Mora M, 
494

495

496

497

498

502

503

504

505

506

507

508

509

510

511

512

513

514

Scarselli M, Ros IM y., Peterson JD, Hauser CR, Sundaram JP, Nelson WC, Madupu R, Brinkac LM, Dodson RJ, Rosovitz MJ, Sullivan SA, Daugherty SC, Haft DH, Selengut J, Gwinn ML, Zhou L, Zafar N, Khouri H, Radune D, Dimitrov G, Watkins K, O’Connor KJB, Smith S, Utterback TR, White O, Rubens CE, Grandi G, Madoff LC, Kasper DL, Telford JL, Wessels MR, Rappuoli R, Fraser CM. 2005. Genome analysis of multiple pathogenic isolates of Streptococcus agalactiae: Implications for the microbial "pan-genome." Proceedings of the National Academy of Sciences of the United States of America 102:1395013955.

Vernikos G, Medini D, Riley DR, Tettelin H. 2015. Ten years of pan-genome analyses. Current opinion in microbiology 23:148-154.

Wyres KL, Wick RR, Judd LM, Froumine R, Tokolyi A, Gorrie CL, Lam MMC, Duchêne S, Jenney A, Holt KE. 2019. Distinct evolutionary dynamics of horizontal gene transfer in drug resistant and virulent clones of Klebsiella pneumoniae. PLoS genetics 15:e1008114.

Yu G, Smith DK, Zhu H, Guan Y, Lam TT. 2017. Ggtree : An r package for visualization and annotation of phylogenetic trees with their covariates and other associated data. Methods in ecology and evolution / British Ecological Society 8:28-36.

Zhang Y, Sievert SM. 2014. Pan-genome analyses identify lineage- and niche-specific markers of evolution and adaptation in Epsilonproteobacteria. Frontiers in microbiology 5:110. 
Figure 1 (on next page)

Flowchart of the Rephine.r pipeline. 

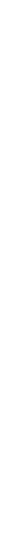

\section{Cluster Merging}

Defragmentation

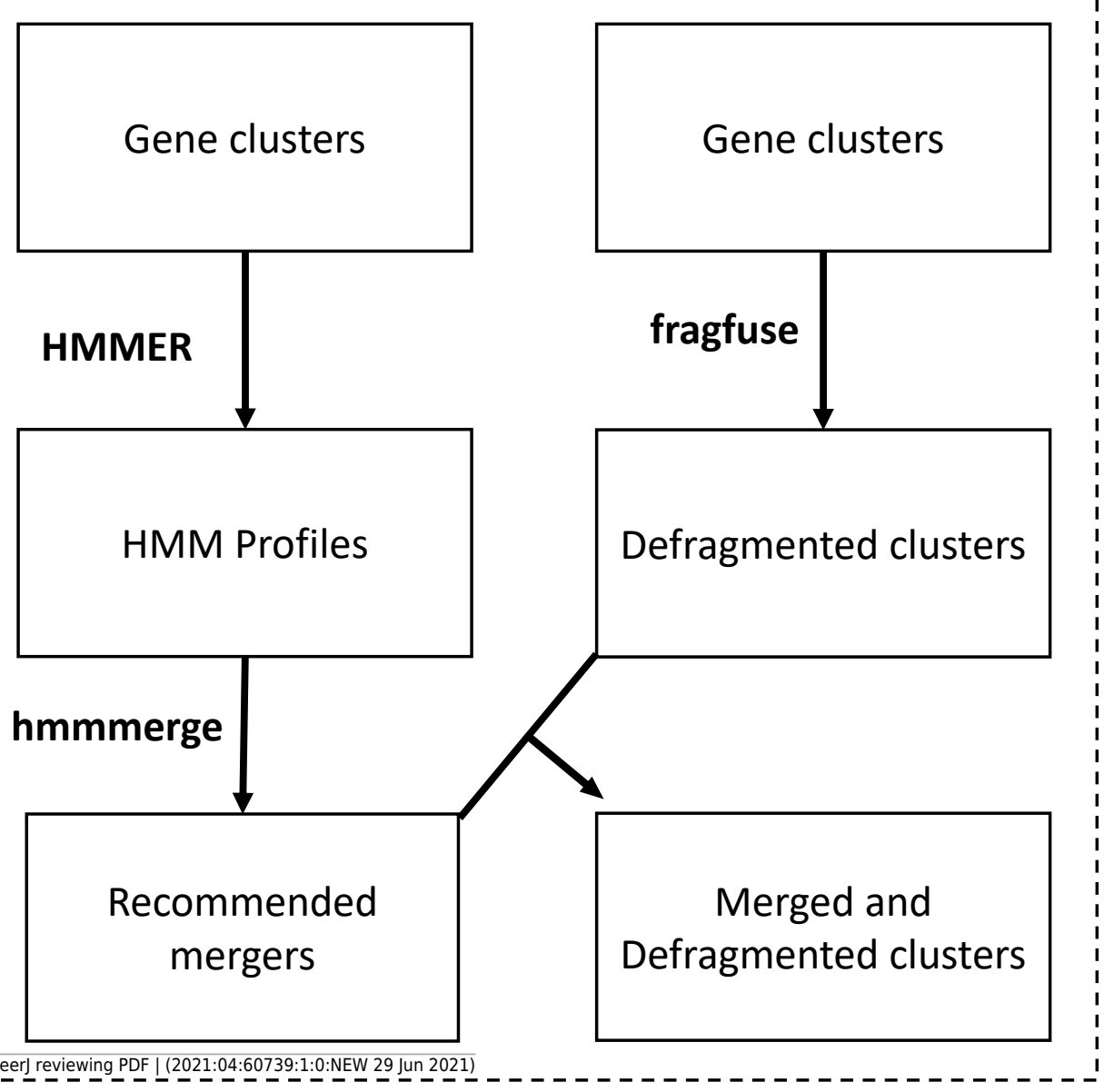




\section{Figure 2}

Fragmented gene calls can be identified from alignments.

(A) An original multiple sequence alignment where the gene from NC_041902 has been split into two fragments by an indel. (B) The corrected alignment following Rephine.r. Highlighted colors are used to indicate regions of each fragment and where they correspond within an intact homolog.

A

$>$ NC_007810_79

MTAKKYYSPDDLVTPQEFADPQFAAINQKRFDLYIDLRVQGYSSWRVFRAIWGEEHMDGPA QARIFAMESNPYYRKQFKAKLNATR TSDLWNPKTALHELLQMVRDPTVKDSSRLSAIKEL NVLAEITFVDESGKTRVGRGLADFYASEAEAQTATVAAAAEANGYVQDGEEGDFPSPTPE PTEEDRANPIQT

$>$ NC $041902 \quad 47$

-MTKKFYSPDDLVTPQEFADPHFAAINQKRFDLYIDLRVQGYSSWRVFRAIWGEEHMDGPA QARIFAMESNPYYRKQFKAKLNATKR----------------- PICGIQRRRST----

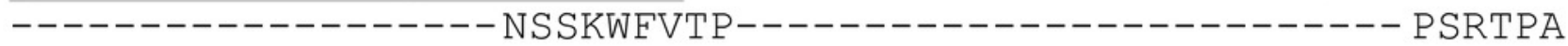
VCRPSRN-----

$>$ NC_041902_46

MVRDPTVKDSSRLSAIKEL NVLAEITFVDESGKTRIGRGLADFYASEAEAQTATVAAAAEANSYVPEGEEGDFPSPTPE PTEEDRANPI--

B

$>\mathrm{NC} \quad 007810 \quad 79$

MTAK̄YYSPDDLVTPQEFADPQFAAINQKRFDLYIDLRVQGYSSWRVFRAIWGEEHMDGPA

QARIFAMESNPYYRKQFKAKLNATR-----------TSDLWN-------PKTALHELLQ MVRDPTVKDSSRLSAIKELNVLAEITFVDESGKTRVGRGLADFYASEAEAQTATVAAAAE ANGYVQDGEEGDFPSPTPEPTEEDRANPIQT

$>\mathrm{NC} \_041902 \_47: 46$

-MTKFYYSPDDLVTPQEFADPHFAAINQKRFDLYIDLRVQGYSSWRVFRAIWGEEHMDGPA QARIFAMESNPYYRKQFKAKLNATKR PICGIQRRRSTNSSKWFVTPPSRTPAVCRPSRN MVRDPTVKDSSRLSAIKELNVLAEITFVDESGKTRIGRGLADFYASEAEAQTATVAAAAE ANSYVPEGEEGDFPSPTPEPTEEDRANPI -- 
Figure 3

Studiervirinae phylogeny before (A) and after (B) using Rephine.r to correct the SCG.

Bootstrap support is shown by coloring branches preceding nodes, with low support (from 0 to 70) ranging from white to red. Increasing the size of the SCG reduced the number of lowsupport branches.
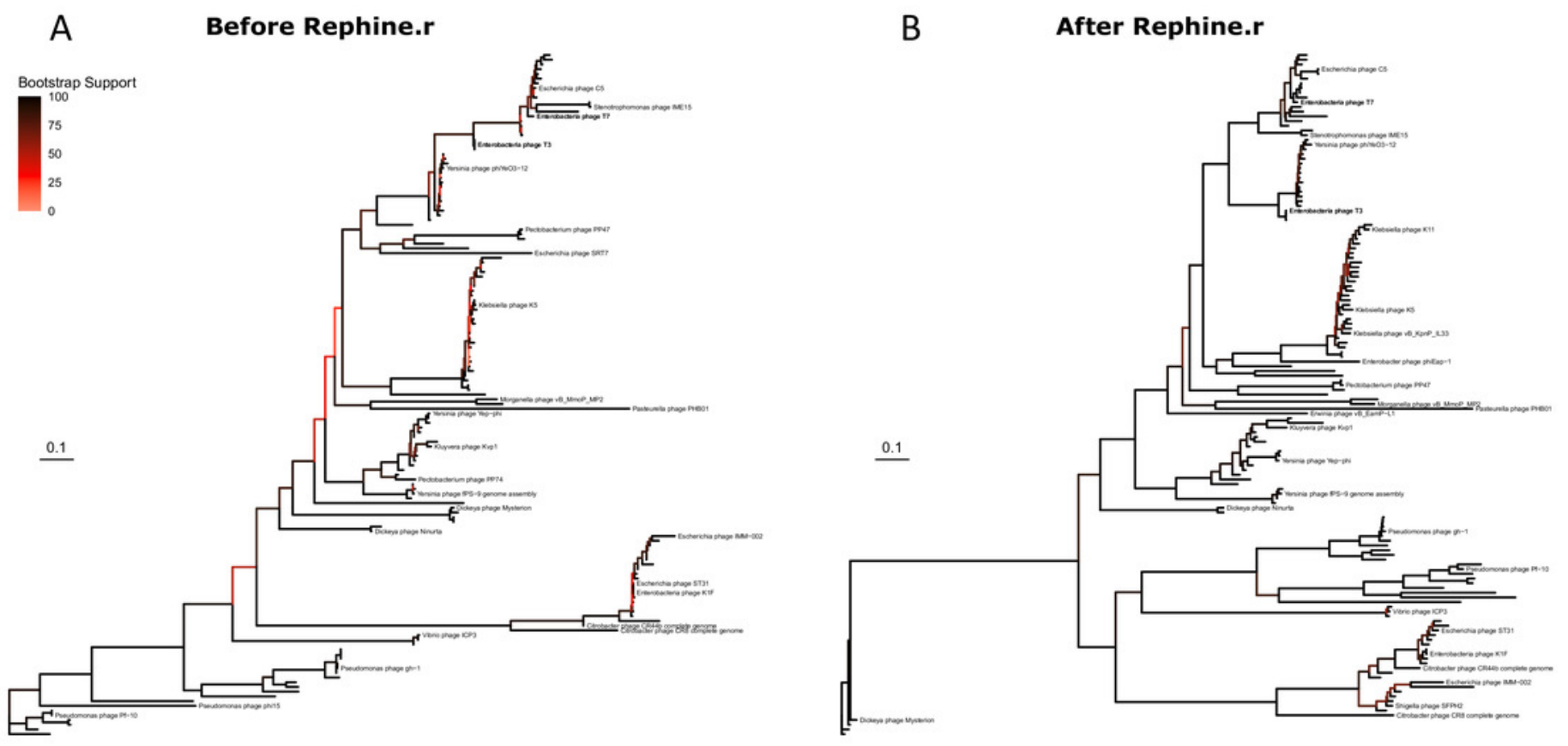


\section{Table 1 (on next page)}

Summary of results of running Rephine.r for each phage group 


\begin{tabular}{r|rrr} 
& Studiervirinae & Tevenvirinae & Pbunaviruses \\
\hline Number of genomes & 145 & 127 & 30 \\
Mean genome size & 39696 & 174775 & 66068 \\
Initial gene calls & 6956 & 35436 & 3540 \\
Initial gene clusters & 558 & 4067 & 195 \\
Initial core genes & 12 & 27 & 28 \\
Initial SCG size & 3 & 13 & 19 \\
New clusters after merging & 16 & 64 & 2 \\
Clusters involved in a merger & 63 & 270 & 5 \\
Biggest merger & 7 & 30 & 3 \\
Core genes after merging & 14 & 37 & 28 \\
SCG size after merging & 3 & 13 & 19 \\
Defragmented clusters & 14 & 99 & 17 \\
SCG size after fusion and merge & 8 & 22 & 26 \\
Additional fusions after merge & 1 & 7 & 1 \\
New core genes after final fusion & 0 & 0 & 0 \\
Total SCG gain & 5 & 9 & 7 \\
Mean tree support before & 77.14 & 87.24 & 63.6 \\
Mean tree support after & 90.55 & 93.44 & 69.57
\end{tabular}

1 